\title{
Does obesity, duration of symptoms and pre-operative knee movements amend discharge readiness and outcomes following total knee arthroplasty?
}

\author{
Thirumal G. Gnaneswaran, Mohamed Nazir Ashik*, Gokul Raj Dhanarajan, Prabaharan C.
}

Department of Orthopedics, Sri Ramachandra Institute of Higher Education and Research, Porur, Chennai, India

Received: 25 October 2021

Revised: 09 November 2021

Accepted: 10 November 2021

\section{*Correspondence:}

Dr. Mohamed Nazir Ashik,

E-mail: dr.ashik2k12@gmail.com

Copyright: () the author(s), publisher and licensee Medip Academy. This is an open-access article distributed under the terms of the Creative Commons Attribution Non-Commercial License, which permits unrestricted non-commercial use, distribution, and reproduction in any medium, provided the original work is properly cited.

\begin{abstract}
Background: Total knee arthroplasty (TKA) being the definite procedure in degenerative arthritis of the knee is associated with a high cost that includes the cost of implants and in-hospital length of stay (LOS). Incurring such high costs will put a burden on the patients economically, so the goal being the reduction of hospital stay yet improving the functional outcome with better patient satisfaction.

Methods: The study design is a retrospective analysis of 1022 patients of primary elective TKA, performed in our institution. The targeted indicators were sex, body mass index (BMI), pre-operative knee range of movements (ROM), knee deformity and duration of symptoms were analyzed.

Results: Analyzing these patients, we found the average LOS is 6.4 days, and obesity, pre-op ROM and deformity all play a role to delay the discharge readiness in the patients. Our study showed that LOS after TKA is multifactorial. Conclusions: In acute setting for readiness of discharge the preoperative knee movements, deformity and patients' functional abilities can be used to segregate patients who may require close monitoring or intensive physiotherapy.
\end{abstract}

Keywords: LOS, BMI, Pre-operative ROM, TKA, Rehabilitation

\section{INTRODUCTION}

Osteoarthritis and rheumatoid arthritis are the important reasons for TKA done and is considered a safe and costeffective procedure that will improve patient's outcomes, quality of life and shrink the pain. ${ }^{1}$ The burden of osteoarthritis has continued to inflate in the society and health care system and this is due to increasing prevalence of degenerative diseases. ${ }^{2,3}$ In countries like India, the developing countries there is always a fear for surgeries and patients worry about the outcome of such replacements and present late to the surgeons. ${ }^{4}$ As the duration of symptoms keep rising, the deformity incurred will be a nightmare for the orthopedic surgeons to provide a favorable outcome for such patients following TKA. The LOS for such patients takes a rise before they have the desired ROM and quadriceps strength. ${ }^{2}$ There is also an increased demand on hospital resources in developing countries to provide adequate care to all patients in terms of beds, and treatment protocols and other professional unit needs that puts a overload on the hospital. Sometimes the patients have to wait for protracted days without getting operated on due to limited resources available. ${ }^{5}$

An increased LOS and undesired outcomes fall to be a burden physically and mentally for such patients, and so they should be previously educated on the expected outcomes. Nonetheless, the importance of these factors on the decision to perform knee arthroplasty is debatable among surgeons. 
The course of action is to dwindle the LOS but still improve the functional outcome with better patient satisfaction. The motive of this study is to identify obesity, the preoperative knee ROM and the duration of symptoms that will amend discharge readiness, by quantifying them, efforts can be done to manage those risks providing better satisfaction to patients, yet lower hospital stays among patients undergoing TKA in a single tertiary care center in India. We hypothesize that several variables will be related to an increase in hospital stay following knee replacement.

\section{METHODS}

A retrospective study was done among patients undergoing unilateral primary total knee replacement at our institute Sri Ramachandra institute of higher education and research from 2012 to 2019 were taken into this study. The inclusion criteria for the selection are all the patients who undergo primary total knee replacement in our institute. The exclusion criteria for this study included patients undergoing revision or multistaged TKA and those with data being unavailable in the institutional electronic database. The focus was on the potential factors consisting of BMI, Duration of the symptoms for which the replacement was done and the pre-operative ROM which will reflect on the postoperative rehabilitation outcomes and all these variables were equated to their length of hospital stay. Institutional ethics committee approval was obtained and sample size was calculated with the help of the statistician with all the data that is available in the medical records department from 2012.

All TKA's were performed by senior-most orthopedic surgeons of our institution. The anesthesia used according the compliance of the patient and anesthetist's choice and a tourniquet was used for almost all patients. As a general practice in our hospital, antibiotic coverage was administered to all patients prophylactically 30 mins to 1 hour before surgery. All TKA's were performed by median parapatellar approach. The use of the implant and the need of drain was by the choice of the surgeon. Routine administration of chemical prophylaxis and mechanical prophylaxis is given to all patients for 2 or 3 days as per their deep vein thrombosis (DVT) risk score to prevent any DVT. Physiotherapy will mobilize the patient with full weight-bearing as tolerated from postoperative day 1 along with quadriceps and hamstring isometric strengthening exercises. Wound inspection and the dressing were done at frequent intervals maintaining sterility. The discharge readiness acts when the patients are met with few norms like Visual analogue score less than 3, walking without support and it includes training them to manage bed to chair transfer, stair climbing and personal care and hygiene. The admitting team will decide the discharge after getting opinion from the rehabilitation team. The objective of the study-the discharge readiness is based on the length of the hospital stay which is defined as the number of days in the hospital from the day of admission to the day of discharge inclusive.

All the details were collected from the institutional medical records department after eliminating the personal details, and our main theme taken into account are the BMI to denote obesity, the duration of symptoms and pre-operative knee movements were included into the statistics. The statistics was done using latest SPSS software and age, deformity, ROM, Duration of symptoms and BMI was equated to the length of hospital stay and the mean, standard deviation and $p$ are determined. In terms of $p$, digits $\mathrm{p} \leq 0.05$ is considered significant and values more than 0.05 is considered insignificant.

\section{RESULTS}

The total number of patients included in study were 1022 out of which 706 female and 316 were male (Figure 1).

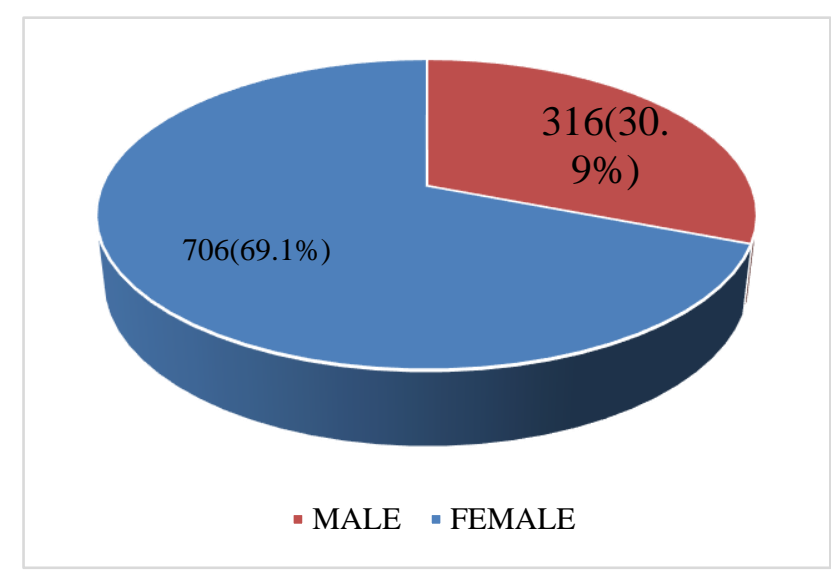

Figure 1: Sex distribution.

The mean duration of hospital stay in male patients was 6.48 days and 6.71 days in females which was found to be insignificant with the $\mathrm{p}=0.32$ (Figure 2).

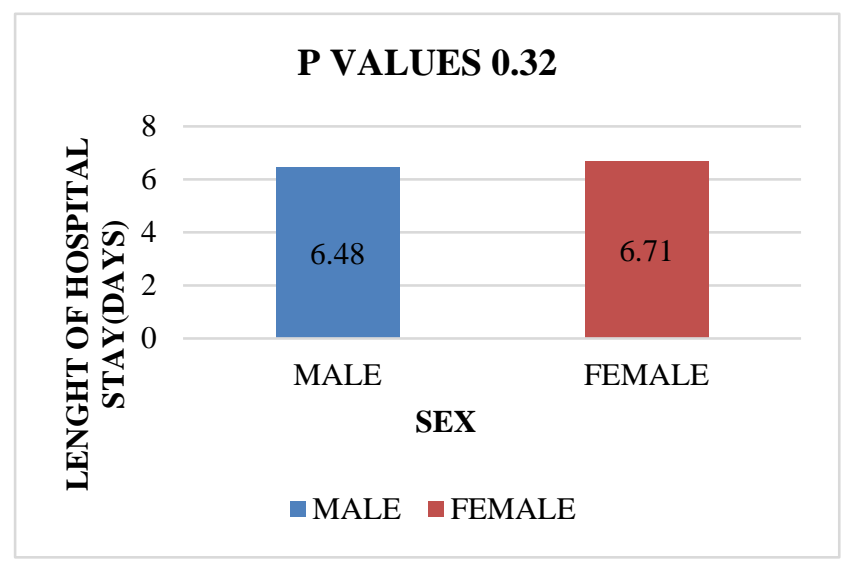

Figure 2: Sex distribution as per duration of hospital stay. 
BMI was calculated for the patients using their weight and height at the time of admission out of 1022 patients only 281 patients have normal BMI (below 24.9). BMI is the direct indicator of the patient's obese status. High BMI leads to difficult rehabilitation and increased risk of DVT with a need for DVT prophylaxis both with mechanical and chemical prophylaxis and increased stay in hospital with a mean hospital stay of 7 days $(\mathrm{p}=0.001)$ (Figure 6).

The duration of symptoms of the patients, the deformity they had pre-operatively and the ROM present preoperatively increase the length of hospital stay thereby changing the discharge readiness of the patients.

Patients with symptoms complaining for more than 10 years have an increased LOS up to 7.42 days whereas the majority of the population having their symptoms less than 10 years and even bare minimum of less than 2 years have a LOS of 6.75 days and 6.43 days respectively with a significant $\mathrm{p}=0.001$ (Figure 3 ).

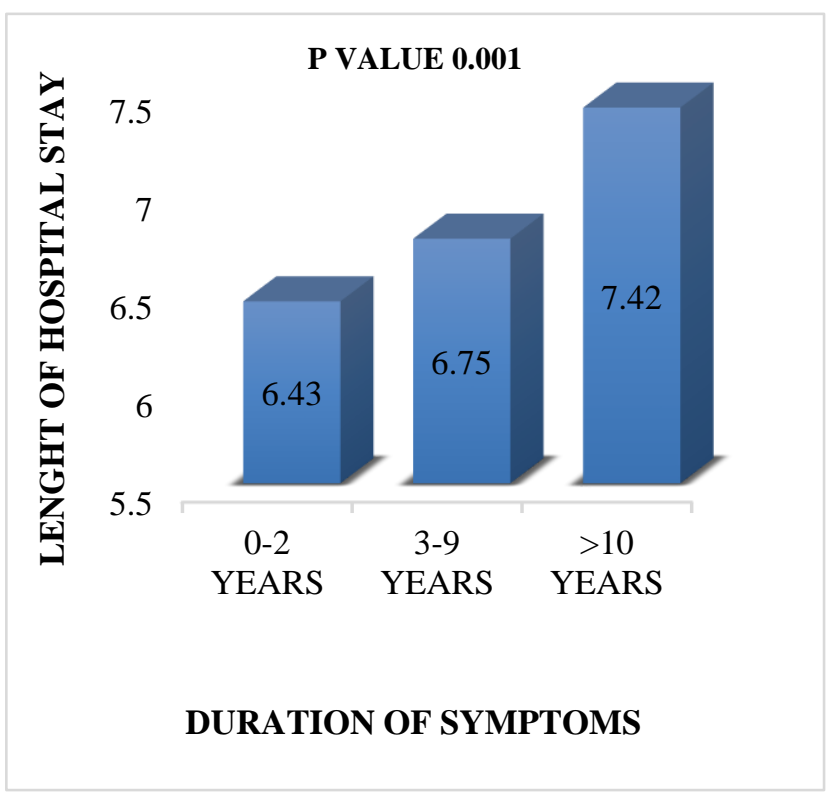

Figure 3: Duration of symptoms.

The patients with lesser ROM and increased deformity of the knees needed extra days of hospital stay before discharge readiness. This is attributed to their increased operative time and so more soft tissue dissection, which may require more days of analgesics and rehabilitation time before the discharge criteria is met. ROM if less than 60 degrees have an extended hospital stay of 8.1 days whereas patients with ROM more than 90 degrees pre-operatively stay an average of 6.1 days with a significant $\mathrm{p}=0.001$. Bulk of patients (836) had less than 10 degrees of varus or valgus deformity during presentation and their average LOS was up to 6.5 days, whereas patients with deformity more than 20 degrees had an in-hospital stay of 7.7 days (Figure 4 and 5).

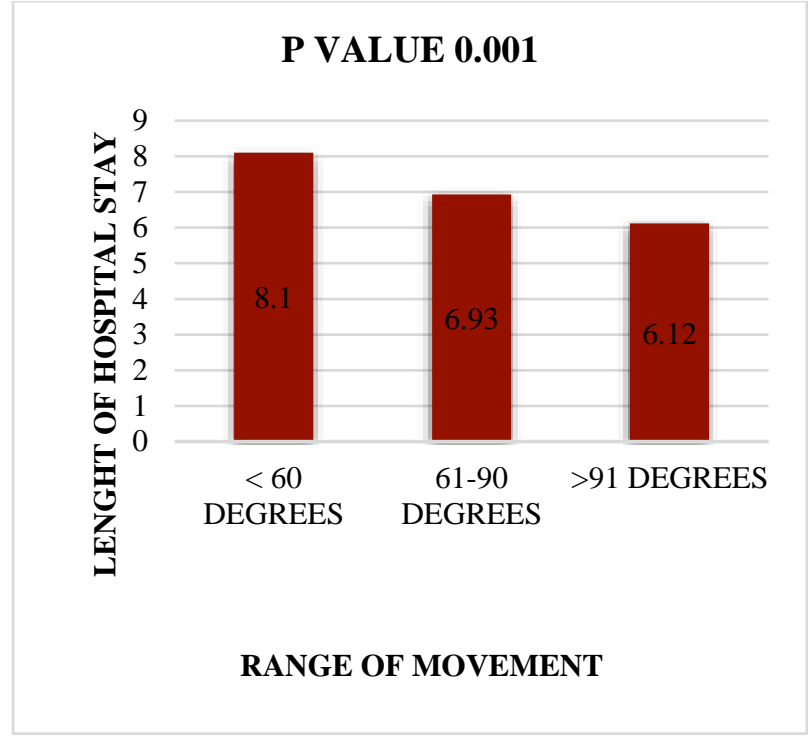

Figure 4: Range of movements.

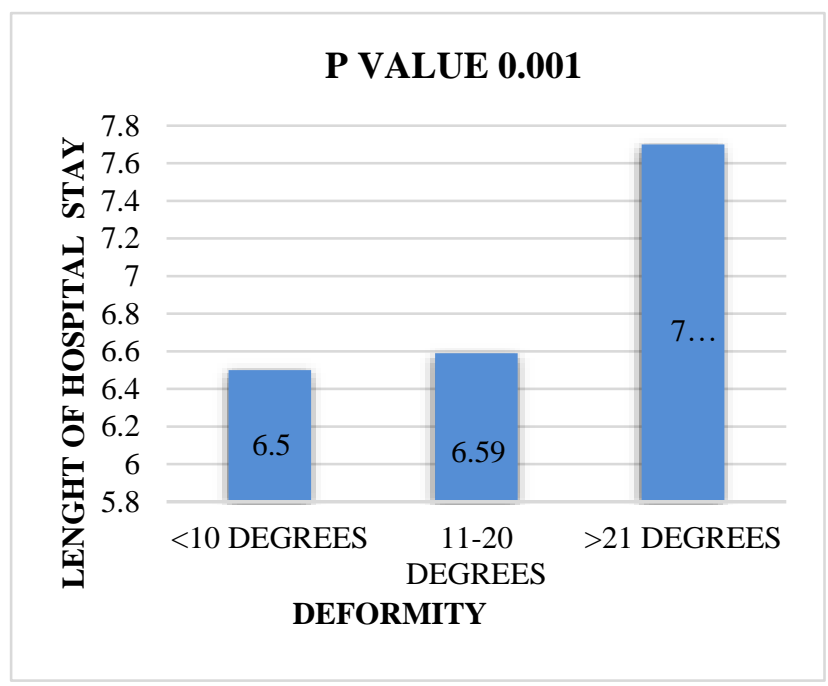

Figure 5: Deformity.

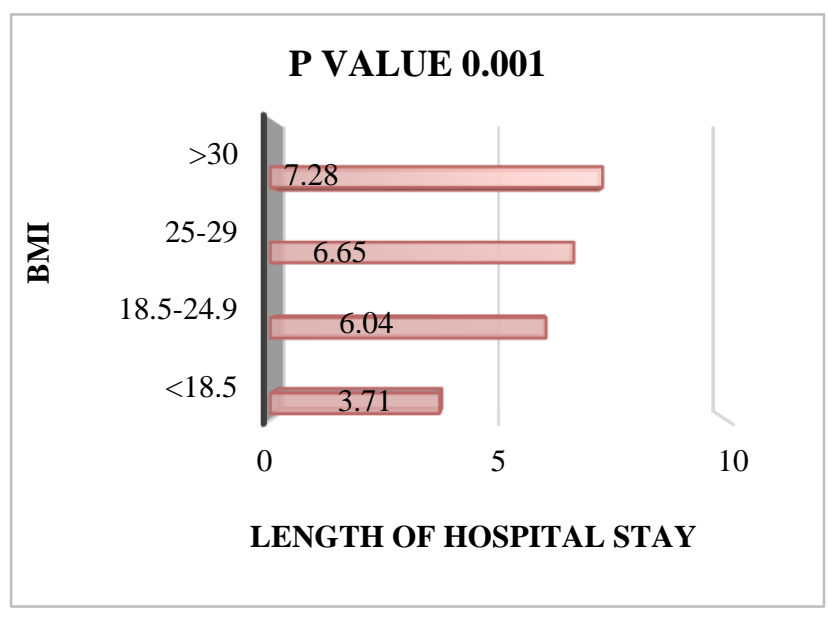

Figure 6: BMI. 
Table 1: Sex, duration of symptoms, ROM, deformity and BMI of participants.

\begin{tabular}{|c|c|c|c|c|}
\hline Variables & Sample, N (\%) & Mean \pm SD & Mean LOS & $P$ value \\
\hline \multicolumn{4}{|l|}{ Sex } & \multirow{3}{*}{0.32} \\
\hline Male & $316(30.9)$ & $6.48 \pm 3.376$ & 6.48 & \\
\hline Female & $706(69.1)$ & $6.71 \pm 3.451$ & 6.71 & \\
\hline \multicolumn{4}{|c|}{ Duration of symptoms (Years) } & \multirow{4}{*}{0.001} \\
\hline $0-2$ & $484(47.4)$ & $6.43 \pm 3.377$ & 6.43 & \\
\hline 3-9 & $484(47.4)$ & $6.75 \pm 3.544$ & 6.75 & \\
\hline More than 10 & $54(5.3)$ & $7.42 \pm 2.678$ & 7.42 & \\
\hline \multicolumn{4}{|c|}{ ROM (Degree) } & \multirow{4}{*}{0.001} \\
\hline$<60$ & $59(5.8)$ & $8.10 \pm 4.217$ & 8.10 & \\
\hline $61-90$ & $509(49.8)$ & $6.93 \pm 3.401$ & 6.93 & \\
\hline$>91$ & $454(44.1)$ & $6.12 \pm 3.428$ & 6.12 & \\
\hline \multicolumn{4}{|c|}{ Deformity (Degree) } & \multirow{4}{*}{0.001} \\
\hline$<10$ & $836(81.8)$ & $6.50 \pm 3.311$ & 6.50 & \\
\hline $11-20$ & $101(9.9)$ & $6.59 \pm 2.748$ & 6.59 & \\
\hline$>21$ & $85(8.3)$ & $7.70 \pm 5.723$ & 7.70 & \\
\hline \multicolumn{4}{|l|}{ BMI $\left(\mathrm{kg} / \mathrm{m}^{2}\right)$} & \multirow{5}{*}{0.001} \\
\hline$<18.5$ & $17(1.7)$ & $3.71 \pm 1.572$ & 3.71 & \\
\hline $18.5-24.9$ & $281(27.5)$ & $6.04 \pm 3.605$ & 6.04 & \\
\hline $25-29$ & $389(38.1)$ & $6.65 \pm 3.218$ & 6.65 & \\
\hline$>30$ & $335(32.8)$ & $7.28 \pm 3.424$ & 7.28 & \\
\hline
\end{tabular}

\section{DISCUSSION}

The outcomes of a well performed TKA, though has numerous grounds, solely depend on the patient's preoperative statuses. Apart from the general health of the patient, the degree of pre-operative movements the knee has, determines the final outcome. To achieve such an outcome, the patient may need an extended hospital stay to impart a disciplined physiotherapy. With increased rates of TKA done in various countries, there is always a continuous motive to reduce the cost of TKA by minimizing the resources yet a better post-operative outcome and better patient satisfaction in a shortened LOS. ${ }^{11,12}$ A proper pre-operative planning and education to the patient to plateau his expectation levels is of utmost importance to reduce the LOS following a primary TKA. BMI which indicates obesity indirectly increases the LOS in our study. The cause attributed is because of an increased operative time and blood loss, forcing a prolonged hospital stay to optimize the blood parameters before readiness to discharge. Gadinsky et al in his study found that obesity was related to longer surgery time in knee replacements. ${ }^{6}$ Nunez et al and Ashik et al in their study found a negative association between BMI and the quality of life after TKR and increased LOS. ${ }^{7,2}$ Few studies have shown no correlation between obesity and increased LOS. ${ }^{8}$

Women stayed longer than men which were consistent in many studies though there is no clear-cut explanation and not statistically significant $(\mathrm{p}=0.32)$ like our study, and some studies found no connection between sex in increased LOS and there is more connection between obesity and osteoarthritis which is common in females than males. ${ }^{5,9}$ Other likelihood is men having more muscle bulk helping in early rehabilitative recovery and early discharge readiness. On the other hand, females have high rates of obesity, postoperative blood transfusion and postoperative complications.

Preoperative ROM of the knee has been the strongest predictor of postoperative ROM aiding the outcomes following TKA, with contributory factors of age, sex, obesity, a history of knee surgery, presence of an extensor lag, diagnosis for which the replacement is done and the technique of surgery done, the soft tissues balanced and the ligament released. ${ }^{10}$ However the ROM at early post-operative period don't detail much on the long term outcomes in terms of knee ROM. Ideally the presence of higher coronal and sagittal plane deformity and a limited pre-operative ROM will help us identify patients who will require an enthusiastic, supervised rehabilitation to get an extra edge of post-operative ROM that will increase the length of hospital stay, that is proven in our study. The longer the duration of symptoms present in patients, will have high levels of pain post operatively due to extensive ligament and soft tissue balancing, increased blood loss and correction of deformity that challenges the patient to start mobilization limit. This also depends on the pain tolerance of the patient, the anesthesia dosage and post-operative analgesia coverage. So, to gain the desired Knee functions, the patient has to stay for longer periods and undergo energetic physiotherapy. This extended stay will however help them gain quadriceps strength but still becomes a burden to the patient financially. The risk factors can be a lectured by reducing the BMI be encouraged for quadriceps and hamstring strengthening exercises to reduce BMI and further help in postoperative rehabilitation. Patients when coming for the first visit 
should be advised to quit substance abuse. Patients should be advised to have a high protein diet if no contraindication is present. All these factors diminish the duration of stay, cost of rehabilitation and postoperative need for mechanical and chemical DVT prophylaxis, thus reducing the cost of stay and burden on patients. The strengths of our study include a large sample size of 1022 patients collected from a single institution and has homogenous operative and postoperative rehabilitation protocol.

\section{Limitations}

Few other confounding factors were not addressed to equate the LOS. Focus was primly on the three above mentioned factors. Also, these values are from the same institution and may not contribute to represent the whole of population.

\section{CONCLUSION}

There are various modifiable and non-modifiable risk factors that are associated with increased length of hospital stay. In acute setting for readiness of discharge the preoperative knee movements, deformity and patients' functional abilities used to segregate patients who may require close monitoring or intensive physiotherapy.

Our study hence concludes saying obesity, preoperative knee ROM and deformity do play a role to increase the length of hospital stay.

\section{ACKNOWLEDGEMENTS}

Author would like to thank Dr. Nithya for the extensive data collection, Ashra for technical support, Dr. Pirai and Swathi doctor for their help in data pooling.

Funding: No funding sources Conflict of interest: None declared

Ethical approval: The study was approved by the institutional ethics committee

\section{REFERENCES}

1. Turki AS, Dakhil YA, Turki AA, Ferwana MS. Total knee arthroplasty: Effect of obesity and other patients' characteristics on operative duration and outcome. World J Orthop. 2015;6(2):284-9.

2. Ashik NM, Qamar A. Factors determining the burden for patients of south Indian population following total knee replacement in terms of hospital stay: A retrospective single center study. J Knee Surg. 2021;34(5):552-60.
3. Scholes C, Cowley M, Ebrahimi M, Genon M, Martin SJ. Factors Affecting Hospital Length of Stay following Total Knee Replacement: A Retrospective Analysis in a Regional Hospital. J Knee Surg. 2021;34(5):552-60.

4. Losina E, Wright EA, Kessler CL, Barrett JA, Fossel $\mathrm{AH}$, Creel $\mathrm{AH}$ et al. Neighborhoods matter: use of hospitals with worse outcomes following total knee replacement by patients from vulnerable populations. Arch Intern Med. 2007;167(2):182-7.

5. Song X, Xia C, Li Q. Perioperative predictors of prolonged length of hospital stay following total knee arthroplasty: a retrospective study from a single center in China. BMC Musculoskelet Disord. 2020;21:62.

6. Gadinsky NE, Manuel JB, Lyman S, Westrich GH. Increased operating room time in patients with obesity during primary total knee arthroplasty: conflicts for scheduling. J Arthroplast. 2012;27:1171-6.

7. Núñez M, Lozano L, Núñez E, Segur JM, Sastre S, Maculé $\mathrm{F}$ et al. Total knee replacement and healthrelated quality of life: factors influencing long-term outcomes. Arthritis Rheum. 2009;61(8):1062-9.

8. Amin AK, Clayton RA, Patton JT, Gaston M, Cook RE, Brenkel IJ. Total knee replacement in morbidly obese patients. Results of a prospective, matched study. J Bone Joint Surg Br. 2006;88(10):1321-6.

9. El Bitar YF, KD, Scaife SL, Horberg JV, Saleh KJ. Hospital Length of Stay following Primary Total Knee Arthroplasty: Data from the Nationwide Inpatient Sample Database. J Arthroplasty. 2015;30(10):1710-5.

10. Bade MJ, Kittelson JM, Kohrt WM, Stevens-Lapsley JE. Predicting functional performance and range of motion outcomes after total knee arthroplasty. Am J Phys Med Rehabil. 2014;93(7):579-85.

11. Prohaska MG. Knee. Pre-operative body mass index and physical function are associated with length of stay and facility discharge after total knee arthroplasty. Knee. 2017;24(3):634-40.

12. Robinson JC, Pozen A, Tseng S, Bozic KJ. Variability in costs associated with total hip and knee replacement implants. J Bone Joint Surg Am. 2012;94:1693-8.

Cite this article as: Gnaneswaran TG, Ashik MN, Dhanarajan GR, Prabaharan C. Does obesity, duration of symptoms and pre-operative knee movements amend discharge readiness and outcomes following total knee arthroplasty? Int J Res Orthop 2022;8:34-8. 